\title{
Extended Rate Equations Model for Describing the Dynamics of the Laser Frequency Spectrum
}

\author{
M. ESKEF* \\ Department of Physics, Atomic Energy Commission of Syria, P.O. Box 6091, Damascus, Syria
}

(Received November 28, 2010; in final form March 30, 2011)

\begin{abstract}
Laser rate equations are written and solved in the frequency domain for homogeneous as well as for inhomogeneous gain. The resulting laser model is capable of describing the fine structure as well as the dynamics of the laser frequency spectrum. The calculation shows that laser lines have a Lorentzian-like lineshape. The linewidth is found to be close to the spectral width of the cavity in case of inhomogeneous gain, whereas it is proved to approach the quantum limit for homogeneous gain.
\end{abstract}

PACS: 42.55.Ah, 42.55.Lt

\section{Introduction}

Laser linewidth plays a key role in manifold applications utilizing laser spectroscopic techniques. The first treatment of laser linewidth was conducted by Schawlow and Townes in the late 1950s. They studied the situation of a single-mode laser with homogeneously broadened gain profile, and found that the emitted laser light should have an extremely small linewidth of the order of a few $\mathrm{Hz}$ which is limited only by means of quantum noise due to spontaneous emission [1]. The quantum theory of laser, developed later on during the early 1960s, improved that picture to include an additional contribution from the so-called technical noise related mainly to fluctuations in the pump power as well as in the effective length of the resonator [2]. Technical noise has been found to yield considerably larger linewidths ranging from a few $\mathrm{kHz}$ up to several tens of MHz. During the late 1960s and early 1970s, great theoretical as well as experimental efforts have been vested into the investigation of laser linewidth due to quantum noise. The Schawlow-Townes quantum limit has been verified to apply precisely in the above threshold region, and within a factor of two when the laser is operated below threshold [3-5]. Since then, it has been taken as a matter of facts that laser linewidth generally approaches the quantum limit, but mostly fails to reach it because of the contribution of technical noise.

Indeed, the picture of infinitely small linewidth has been adopted in the manifold of laser models developed during the past decades, both semiclassical $[6,7]$ as well as quantum statistical ones $[8,9]$. However, in all those models, the vanishing linewidth was not a physical result of the homogeneous gain as in the theoretical study of Schawlow and Townes, but rather an artificial result of requiring the solutions for the field to be self-consistent. Let us note that the latter requirement itself is less convincing since it fails to explain the frequency spectrum of the pure cavity.

Therefore, and notwithstanding the great significance of the existing laser models regarding the investigation

* e-mail: pscientific1@aec.org.sy of multitude applications, we believe that there is still a need for a laser model which re-establishes the missing connection between the mechanism responsible for fine-structuring the laser frequency spectrum and the elementary process of stimulated emission. On the other hand, stimulated emission is directly coupled to the dynamics of population inversion, and the latter is known to show completely different behaviour for homogeneous and inhomogeneous gain profile $[10,11]$. For this reason, it is desirable to start with studying the two situations, homogeneous and inhomogeneous gain, separately before proceeding to consider the general situation of a gain profile with both homogeneous and inhomogeneous contributions. The present work is devoted to exposing these problems on the basis of an extended rate equations model.

Laser models based on Einstein's rate equations have proved to be quite powerful in describing the dynamics of the interplay between pumping, laser power, and population inversion [12-16]. In their conventional form, laser rate equations are written and solved in the pure time domain, and include no information about the detailed structure of the frequency spectrum. In order to make the rate equations capable of describing the dynamics of the laser frequency spectrum, two steps should be done. Firstly, the rate equations should be extended into the frequency domain; and secondly, the spectral effect of the cavity should be adequately incorporated into the extended rate equations.

The deduction and discussion of the extended rate equations for homogeneous, as well as for inhomogeneous gain, constitute the main subject of the present study. We will rely on gas lasers in order to compare our calculation results with the experimental data available from our $\mathrm{He}-\mathrm{Ne}$ laser. However, the basic arguments of extending the laser rate equations into the frequency domain should apply equally for other kinds of lasers.

\section{The model}

A laser consists, in general, of three fundamental components: an active medium providing the spontaneous emission (source) as well as the stimulated emission (am- 
plification), a pumping mechanism supporting the population of the laser upper state, and an optical resonator providing the feedback to the active medium. Within the framework of a three-level formalism which is quite sufficient for the purpose of the present study, the active medium is characterized by the population densities $M_{1}$ and $M_{2}$ of the laser lower, respectively upper state, the decay constants $A_{10}$ and $A_{20}$ describing the nonradiative decay of the lower, respectively upper state into the ground state, the Einstein coefficient $A_{21}$ accounting for the spontaneous decay of the upper into the lower state, and the cross-section $\sigma$ for the stimulated transition $1 \rightarrow 2$ (absorption), as well as $2 \rightarrow 1$ (stimulated emission). The pumping mechanism, second component of the laser, is simply described by the pumping rate $\Phi$. The resonator, third component of the laser, is specified as a Fabry-Perot cavity characterized by its optical length $L$ and the mirror reflectivities $R_{1}$ and $R_{2}$.

In constructing our rate equations, we will follow the outlines of the common concept used by Burak et al. for small gain lasers [17], as well as by Rigrod [18] and Kaufman and Oppenheim [19] for high gain lasers. However, our treatment will distinguish itself from earlier works by means of the following two additional features. (a) We will write and solve the laser rate equations in both time and frequency domains; and (b) we will introduce the spectral effect of the cavity into the laser rate equations instead of adopting predefined cavity modes. These two add-ons will give our extended model the capability of providing an independent calculation of the time evolution of the laser frequency spectrum in contrast to conventional rate equations models which are restricted to the dynamics of a few predefined modes. In the following, we will distinguish between two idealized situations specified by a completely inhomogeneous, respectively a completely homogeneous gain.

\subsection{Inhomogeneous gain}

In this idealized situation, the frequency spectrum of the source photons (spontaneous emission) is assumed to be inhomogeneously broadened by means of the Doppler broadening. Under this assumption, the atoms/ molecules of the active medium can be divided into distinct velocity groups associated with different frequency bins. This gives us the opportunity to write and solve the rate equations for each frequency bin separately. Therefore, our rate equations will involve the differential quantities $M_{1}^{\prime}(\nu), M_{2}^{\prime}(\nu), \Phi^{\prime}(\nu)$, and $\beta^{\prime}(\nu)$ which describe the population densities, the pumping rate, and the rate of stimulated emission for the group of atoms/molecules associated with the frequency $\nu$.

The set of rate equations consists of two equations for the rate of change of the population density of the lower, respectively the upper laser state, as well as a third equation describing the rate of change of the beam intensity inside the cavity. Making use of the differential quantities defined above, the rate equations at frequency $\nu$ can be written as follows:

$$
\begin{aligned}
& \frac{\mathrm{d}}{\mathrm{d} t} M_{1}^{\prime}(\nu)=\beta^{\prime}(\nu)+A_{21} M_{2}^{\prime}(\nu)-A_{10} M_{1}^{\prime}(\nu), \\
& \frac{\mathrm{d}}{\mathrm{d} t} M_{2}^{\prime}(\nu)=-\beta^{\prime}(\nu)-\left(A_{21}+A_{20}\right) M_{2}^{\prime}(\nu)+\Phi^{\prime}(\nu), \\
& \frac{\mathrm{d}}{\mathrm{d} t} I(\nu)=\frac{1}{\tau}\left[R_{1} R_{2} G^{2}(\nu)-1\right] I(\nu),
\end{aligned}
$$

where $\tau=2 L / c$ is the round-trip time and $G(\nu)$ is the single-pass gain due to stimulated emission. Equations (1), (2) are self-explanatory. Equation (3) can be obtained by considering the change of the beam intensity during the round-trip time $\tau$.

Equations (1)-(3) involve five unknown quantities and hence, we still have to construct two additional independent equations of the same quantities in order to achieve a complete set of equations with unique solution. We will do this in the following by scrutinizing closely the single pass amplification $G(\nu)$ as well as the rate of stimulated emission $\beta^{\prime}(\nu)$.

When the beam passes an infinitesimal length $\mathrm{d} l$ inside the active medium, the beam intensity increases by

$$
\mathrm{d} I(\nu)=\sigma_{\operatorname{inh}}(\nu)\left[M_{2}^{\prime}(\nu)-M_{1}^{\prime}(\nu)\right] I(\nu) \mathrm{d} l,
$$

where $\sigma_{\text {inh }}(\nu)$ refers to the effective inhomogeneous transition cross-section. The integration over the full path $l$ throughout the active medium gives the following relationship between outgoing and incoming intensities:

$$
I_{\text {out }}(\nu)=I_{\text {in }}(\nu) \exp \left(\sigma_{\text {inh }}(\nu)\left(\left[M_{2}^{\prime}(\nu)-M_{1}^{\prime}(\nu)\right] l\right) .\right.
$$

Let us note that the latter integration is based on the assumption that changes in $M_{1}^{\prime}$ and $M_{2}^{\prime}$ over the time interval $l / c$ are relatively small (rate equations approximation). The latter relationship provides an intuitive and quite powerful description of the amplification due to stimulated emission $\left(M_{2}^{\prime}>M_{1}^{\prime}\right)$ as the physical counterpart of the attenuation due to absorption $\left(M_{1}^{\prime}>M_{2}^{\prime}\right)$. Moreover, it allows for specifying the single pass amplification $G(\nu)$, also referred to as single pass gain, according to

$$
G(\nu)=\exp \left(\sigma_{\operatorname{inh}}(\nu)\left[M_{2}^{\prime}(\nu)-M_{1}^{\prime}(\nu)\right] l\right) .
$$

Herein, $\sigma_{\operatorname{inh}}(\nu)$ distinguishes itself from the free transition cross-section $\sigma$ by including the frequency selective effect of the cavity. In fact, the quantum electrodynamics tells us that the transition amplitude is proportional to the amplitude of the electromagnetic wave associated with the emitted photon and hence, the transition probability is proportional to the intensity [20]. However, a photon with frequency $\nu$ propagating inside the cavity will undergo single photon interference [21], and this will lead to an intensity enhancement proportional to the Airy function of the cavity. Therefore, the effective cross-section $\sigma_{\text {inh }}(\nu)$ can be written as

$$
\sigma_{\text {inh }}(\nu)=\sigma \frac{1}{1+R_{1} R_{2}-2 \sqrt{R_{1} R_{2}} \cos \delta(\nu)},
$$

where $\delta(\nu)=4 \pi L \nu / c$ is the round-trip phase shift.

On the other hand, the beam entering the active medium from the side of mirror 1 with an intensity 
$I(\nu)$ will exit the medium with the amplified intensity $G(\nu) I(\nu)$, i.e. with an intensity change of $[G(\nu)-1] I(\nu)$. After that, the beam will be reflected on mirror 2 to re-enter the medium with the intensity $R_{2} G(\nu) I(\nu)$ and leave it with the amplified intensity $R_{2} G^{2}(\nu) I(\nu)$, i.e. with an additional intensity change of $[G(\nu)-$ $1] R_{2} G(\nu) I(\nu)$. And hence, the total intensity change due to stimulated emission during a round-trip is given by

$$
\Delta I_{\mathrm{st}}(\nu)=[G(\nu)-1]\left[1+R_{2} G(\nu)\right] I(\nu),
$$

and the rate of stimulated emission $\beta^{\prime}(\nu)$ can be calculated as

$$
\beta^{\prime}(\nu)=\frac{1}{h \nu l}[G(\nu)-1]\left[1+R_{2} G(\nu)\right] I(\nu) .
$$

With the aid of Eqs. (4), (5), the set of rate Eqs. (1)-(3) can be solved for each frequency bin separately. The obtained solution provides the time evolution of the spectral intensity $I(\nu)$, as well as of the population densities $M_{1}^{\prime}(\nu)$ and $M_{2}^{\prime}(\nu)$.

\subsection{Homogeneous gain}

Under the assumption of a homogeneous gain, the atoms/molecules in the lower, respectively the upper state are all equivalent regarding their interaction with photons. Therefore, the population densities $M_{1}$ and $M_{2}$ are no more frequency dependent. Consequently, the pumping rate $\Phi$ and the rate of stimulated emission $\beta$, which directly contribute to the rate of change of $M_{1}$ and $M_{2}$, are also not frequency dependent. Therefore, the rate equations for $M_{1}$ and $M_{2}$ reduce to the simple form

$$
\begin{aligned}
& \frac{\mathrm{d}}{\mathrm{d} t} M_{1}=\beta+A_{21} M_{2}-A_{10} M_{1}, \\
& \frac{\mathrm{d}}{\mathrm{d} t} M_{2}=-\beta-\left(A_{21}+A_{20}\right) M_{2}+\Phi .
\end{aligned}
$$

The rate equation for the intensity keeps the same form given in Eq. (3) and will be written here just for the purpose of completeness

$$
\frac{\mathrm{d}}{\mathrm{d} t} I(\nu)=\frac{1}{\tau}\left[R_{1} R_{2} G^{2}(\nu)-1\right] I(\nu) .
$$

The equation for the single pass gain $G(\nu)$ can be rewritten as

$$
G(\nu)=\exp \left(\sigma_{\mathrm{h}}(\nu)\left(M_{2}-M_{1}\right) l\right) .
$$

Herein, $\sigma_{\mathrm{h}}(\nu)$ refers to the effective homogeneous transition cross-section which can be represented as a Lorentzian-shaped cross-section multiplied by the Airy function of the cavity

$$
\begin{gathered}
\sigma_{\mathrm{h}}(\nu)=\sigma \frac{1}{2 \pi} \frac{\Delta \nu_{\mathrm{h}} \mathrm{d} \nu}{\left(\nu-\nu_{0}\right)^{2}+\frac{1}{4}\left(\Delta \nu_{\mathrm{h}}\right)^{2}} \\
\times \frac{1}{1+R_{1} R_{2}-2 \sqrt{R_{1} R_{2}} \cos \delta(\nu)},
\end{gathered}
$$

where $\nu_{0}$ is the central frequency and $\Delta \nu_{\mathrm{h}}$ the homogeneous width.

The rate of stimulated emission $\beta$ can be calculated by summing up Eq. (5) over all frequency bins

$$
\beta=\sum_{\nu} \frac{1}{h \nu l}[G(\nu)-1]\left[1+R_{2} G(\nu)\right] I(\nu) .
$$

Finally, the complete set of Eqs. (6)-(10) can be solved by means of numerical integration. The solution provides the time evolution of $I(\nu), M_{1}$ and $M_{2}$.

\section{Results and discussion}

In the following, we will discuss the results produced by the model under the conditions of inhomogeneous, respectively homogeneous gain. An overview of the adopted values for the various model parameters is given in Table. The values of the laser parameters are adjusted to reproduce the parameters of our $\mathrm{He}-\mathrm{Ne}$ laser in order to allow for comparison with experiment. The lifetimes $T_{1}$ and $T_{2}$ are taken from [22]; the Einstein coefficient $A_{21}$ and the laser transition cross-section $\sigma$ are taken from [23] and [24], respectively; whereas the value for the pumping rate $\Phi$ is derived from the inversion density of $1.5 \times 10^{9} \mathrm{~cm}^{-3}$ reported in [22].

TABLE

The model parameters adopted for simulating our He-Ne laser.

\begin{tabular}{l|c|l}
\hline \hline Parameter & & Value \\
\hline cavity length & $L$ & $77 \mathrm{~cm}$ \\
mirror reflectivities & $R_{1}$ & 0.99 \\
& $R_{2}$ & 0.98 \\
$\begin{array}{c}\text { length of the } \\
\text { discharge tube }\end{array}$ & $l$ & $60 \mathrm{~cm}$ \\
$\begin{array}{c}\text { lifetime of the laser } \\
\text { upper level }\end{array}$ & $T_{2}$ & $100 \mathrm{~ns}$ \\
$\begin{array}{l}\text { lifetime of the laser } \\
\text { lower level }\end{array}$ & $T_{1}$ & $10 \mathrm{~ns}$ \\
$\begin{array}{c}\text { Einstein coefficient } \\
\text { for the spontaneous } \\
\text { transition 2 } \rightarrow 1\end{array}$ & $A_{21}$ & $3.4 \times 10^{6} \mathrm{~s}^{-1}$ \\
$\begin{array}{c}\text { cross-section } \\
\text { for stimulated } \\
\text { transition } 1 \leftrightarrow 2\end{array}$ & $\sigma$ & $3.0 \times 10^{-13} \mathrm{~cm}^{2}$ \\
pumping rate & $\Phi$ & $1.5 \times 10^{16} \mathrm{~cm}^{-3} \mathrm{~s}^{-1}$ \\
\end{tabular}

At the beginning of the calculation, the system is initialized to meet the conditions of pumping saturation in the open resonator (equilibrium between pumping and decay considering the stimulated emission to be turned off for the time being). Starting from this well defined initial situation, we turn on stimulated emission, and continue tracing the time evolution of the spectral intensity $I(\nu)$ as well as of the population densities $M_{1}$ and $M_{2}$ until saturation is completely established.

\subsection{Inhomogeneous gain}

We will rely on the specific situation of inhomogeneous gain due to the Doppler broadening which is most relevant for the purpose of simulating our $\mathrm{He}-\mathrm{Ne}$ laser aiming at a reliable comparison between calculation and exper- 
iment. Accordingly, we assume the pumping rate $\Phi^{\prime}(\nu)$, the initial population densities $M_{1}^{\prime}(\nu)$ and $M_{2}^{\prime}(\nu)$, as well as the initial intensity (spontaneously emitted photons) to follow a Gaussian distribution. The Doppler width is set to $1500 \mathrm{MHz}$ which is typical for a He-Ne laser oscillating at $\lambda=632.8 \mathrm{~nm}$ [10].

The comparison between calculation and experiment proves, first of all, that our simple model is able to reproduce the experimentally measured frequency spectrum with respect to the number of active modes and their individual positions and relative intensities as readily seen in Fig. 1. However, the calculated overall output power is found to underestimate the measured power of $6.4 \mathrm{~mW}$ by a large factor of several hundreds. Note that the calculated spectrum has been scaled by a factor of $3.7 \times 10^{2}$ in order to fit together with the measured spectrum within the same graph in Fig. 1.

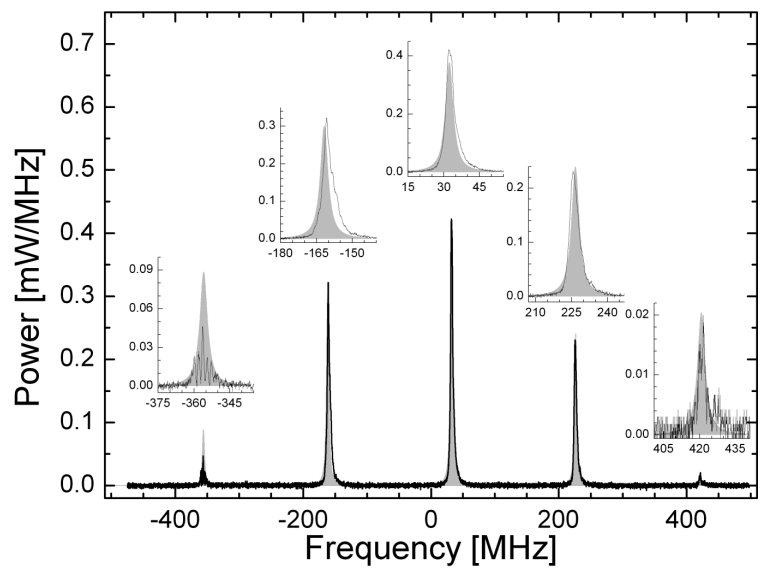

Fig. 1. Frequency spectrum of the He-Ne laser: Comparison between calculation and experiment. The solid line represents the measured spectrum as recorded by a scanning Fabry-Perot interferometer with a resolution of $3.9 \mathrm{MHz}$. The filled area represents the calculated spectrum passed through the Fabry-Perot analyzer and scaled by a factor of $3.7 \times 10^{2}$. The calculation is carried out using the parameter set listed in Table and assuming an inhomogeneous gain with a Doppler width of $1500 \mathrm{MHz}$.

It is worth highlighting the physical content of these seemingly contradictory results before proceeding to further aspects of the comparison between calculation and experiment. Actually, the reproduction of the number of active modes and their positions and relative intensities simply indicates that the model correctly reconstructs the threshold condition for each individual frequency bin. However, the strongly underestimated power exposes the problem connected with our too hard assumption of completely inhomogeneous gain which is hardly applicable for a real He-Ne laser. In fact, the assumption of a completely inhomogeneous gain implies that the total homogeneous width (natural width, pressure broadening, saturation broadening...) is smaller than the cavity width; and this is by far not fulfilled for real He-Ne

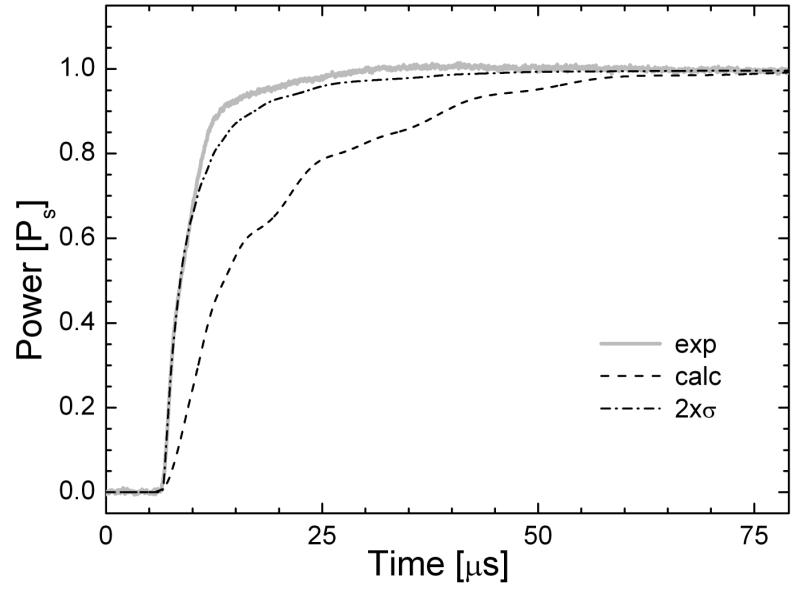

Fig. 2. Comparison between measured and calculated dynamics of the laser power. The solid line shows the experimentally measured rise curve of the power, the dashed line represents the theoretical rise curve calculated with the parameter set of Table and assuming an inhomogeneous gain with a Doppler width of $1500 \mathrm{MHz}$. The dashed-dotted line represents a recalculation with an effective transition cross section of twice the value given in Table. The values of the laser power are given in units of the corresponding saturation power.

lasers. Note that the natural width of the laser transition at $\lambda=632.8 \mathrm{~nm}$ amounts to $19 \mathrm{MHz}$ [10] which clearly exceeds the typical cavity width of about $1 \mathrm{MHz}$. As consequence of the completely inhomogeneous gain, photons with a certain frequency $\nu$ are amplified in the calculation exclusively by the gain available in the corresponding frequency bin whereas in a real He-Ne laser, amplification is massively enhanced by contributions from adjacent frequencies within the homogeneous width. The right way to resolve this power discrepancy is to incorporate the effect of both homogeneous and inhomogeneous broadening of the gain profile into the set of rate equations. In fact, it is not possible to neutralize the power discrepancy within the framework of our model in its present form. The obvious attempt to enhance the power by tuning $\sigma$ or $\Phi$ to larger values would necessarily cause a shift in the threshold balance leading to the activation of additional modes at the cost of the relative intensities of the central ones. By way of illustration, assigning $\sigma$ an artificial value of twice the value listed in Table increases the power by factor 10 , but produces at the same time four additional modes causing the central mode to lose $35 \%$ of its relative intensity.

Concerning the lineshape and linewidth of the individual modes, the calculation produces a Lorentzian-like lineshape and a linewidth close to the spectral width of the pure cavity. The lineshape differs from an ordinary Lorentzian by having a strongly suppressed tail. A direct comparison with the lineshape and linewidth of the experimentally measured frequency spectrum is unfortunately not relevant since the apparent linewidth of 
$3.9 \mathrm{MHz}$ seen in Fig. 1 for both the measured as well as the calculated spectrum does mainly reflect the spectral resolution of the scanning Fabry-Perot interferometer used in the measurement. Note that the calculated spectrum has been passed through the Fabry-Perot in order to enable for a reliable comparison between calculation and experiment. Likewise, it is also not reasonable to compare our calculated linewidth with the experimental results of earlier works reporting very small linewidths of the order of a few $\mathrm{kHz}[5,25]$ because our calculation is based on assuming a completely inhomogeneous gain, which is not reliable for real He-Ne lasers. In fact, we do expect our calculated linewidth to decrease dramatically when allowing for frequency competition due to homogeneous broadening as will be discussed below.

Proceeding to the next item of assessing the results of the calculation in the light of the available experimental data, the calculated time evolution of the power $P(t)$ is shown in Fig. 2 (dashed line) in direct comparison with the rise curve of our $\mathrm{He}-\mathrm{Ne}$ laser (solid gray line) measured by means of a fast photodiode and an oscilloscope with a time resolution of $5 \times 10^{-11} \mathrm{~s}$.

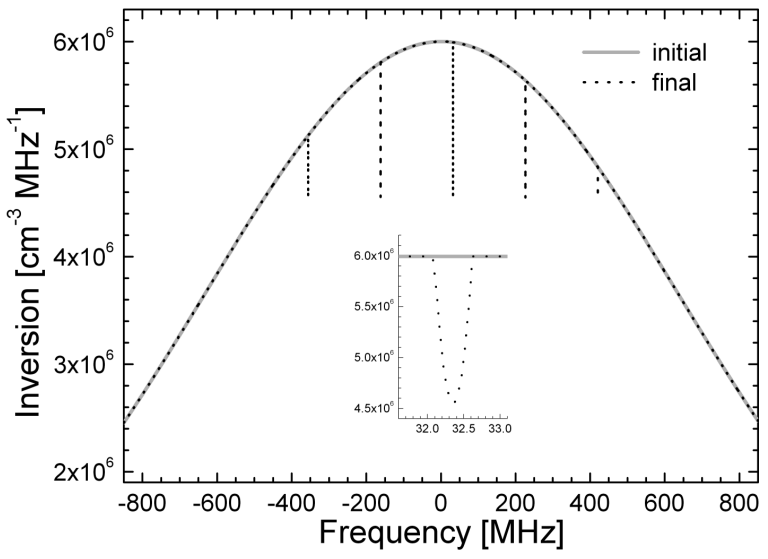

Fig. 3. Spectral distribution of the population inversion calculated for inhomogeneous gain with a Doppler width of $1500 \mathrm{MHz}$. The calculation is carried out using the parameter set of Table. The final inversion (dotted line) deviates from the initial inversion (solid line) only at the positions of the five active modes. A closer view of the population inversion at the position of the most central mode is shown in the inset.

It is evident from Fig. 2 that the calculation qualitatively reproduces the saturation which is the most essential feature of the rise curve. However, a satisfactory reproduction of the rise time is not possible, unless $\sigma$ is artificially enhanced (dashed-dotted line) at cost of producing additional active modes. And this illustrates that the problem of neglecting the homogeneous width in the calculation causes not only a decrease of the power but also an increase of the power rise time.

Completing the discussion of inhomogeneous gain, a quick glance at the calculated population inversion is given in Fig. 3. It is obvious that the saturated pop-

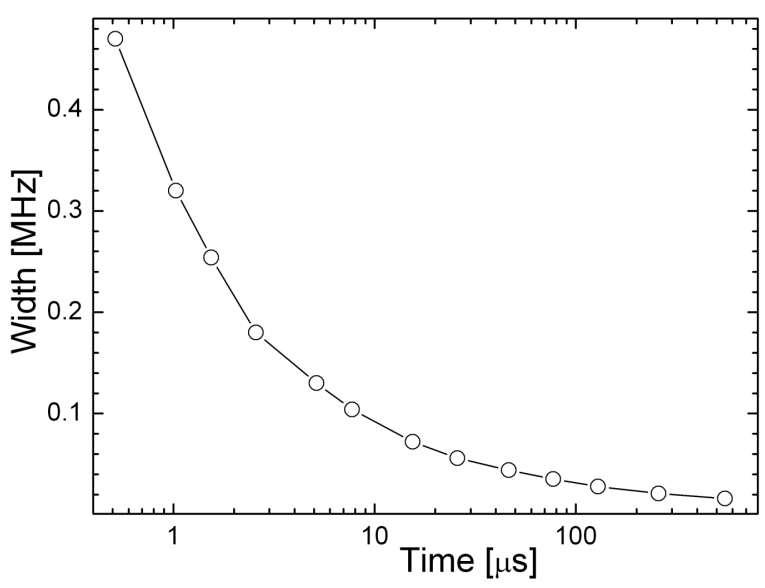

Fig. 4. Dynamics of the laser linewidth for homogeneously broadened gain. The calculation is carried out using the parameter set of Table assuming a homogeneous gain with a Lorentzian width of $30 \mathrm{MHz}$.

ulation inversion (dotted line) differs from the initial inversion (solid gray line) only by means of a few narrow dips at the positions of the active modes, which is quite in line with the common picture for lasers with inhomogeneous gain [10, 11]. A closer look (inset in Fig. 3) shows that the width of the individual inversion dips follows the spectral width of the pure cavity likewise with the linewidth of the corresponding modes. However, this result depends again on our assumption of completely inhomogeneous gain, i.e. on turning off the effect of frequency competition. In fact, we expect, when allowing for frequency competition within a reasonable homogeneous width larger than the cavity width, that the width of the inversion dips would increase towards the homogeneous width whereas the linewidth of the individual modes would decrease to smaller values far below the cavity width.

\subsection{Homogeneous gain}

Now, having disputed the situation of completely inhomogeneous gain, it is worth making a side trip to the complementary situation of completely homogeneous gain. The laser parameters are kept at their values, whereas the gain profile is switched to homogeneous broadening with a moderate width of $30 \mathrm{MHz}$ which is slightly above the natural width of the laser transition. Although no more directly comparable with our He-Ne laser, calculation under these new conditions is of great significance because of several reasons. Firstly, it can be considered to simulate a single mode $\mathrm{He}-\mathrm{Ne}$ laser which is achievable by adjusting the pumping rate to a near-threshold value, i.e. by suppressing the width of the above threshold region to a value smaller than the mode spacing. Secondly, the homogeneous broadening can never be turned off in a real laser; moreover, it clearly exceeds the cavity width in mostly all common lasers and therefore, it is expected to affect the lineshape and linewidth even in case 
of a multimode laser with predominantly inhomogeneous gain. Finally, the homogeneous broadening determines the range of frequency competition which is expected to play a key role in constructing the fine structure of the laser frequency spectrum.

We have calculated the laser frequency spectrum under the assumption of a homogeneous gain with a Lorentzian width of $30 \mathrm{MHz}$. Regarding the overall power, saturation is reached already after a few hundreds round-trips $(1 \mu \mathrm{s})$. The corresponding frequency spectrum is characterized by a single mode coinciding with the strongest available cavity mode, a Lorentzian lineshape, and a linewidth close to the spectral width of the cavity. However, and notwithstanding the rapid saturation of the overall power, the linewidth remains unsaturated after $10^{5}$ round-trips ( $\left.c a .500 \mu \mathrm{s}\right)$ and continues to decrease with time approaching the calculation limit of a single frequency bin $(10 \mathrm{kHz})$ as illustrated in Fig. 4. This is quite in line with the very small quantum limited width predicted by Schawlow and Townes [1]. Let us note that the quantum limit of the linewidth is, in case of our $\mathrm{He}-\mathrm{Ne}$ laser, of the order of $10^{-3} \mathrm{~Hz}$ which is simply unreachable within the framework of our numerical calculation.

\section{Conclusions}

We have demonstrated the possibility of extending the rate equations model into the frequency domain aiming at describing the dynamics of the laser frequency spectrum. Laser rate equations have been written in the frequency domain under the condition of completely inhomogeneous, as well as completely homogeneous broadening of the gain profile. The spectral effect of the cavity has been incorporated into the rate equations by considering the relationship between the probability for stimulated transitions and the cavity-sensitive intensity enhancement due to single-photon interference. Our extended rate equations model proves to provide a reliable tool for investigating the dynamics of the laser frequency spectrum, in contrast to conventional rate equations models which are restricted to describing the dynamics of the integrated photon densities in a few predefined modes.

The validity of the model has been verified by means of comparison between calculation and experiment. Adopting the parameters of our He-Ne laser and assuming an inhomogeneous gain with a Doppler width of $1500 \mathrm{MHz}$, the calculation proved to satisfactorily reproduce the experimentally measured frequency spectrum regarding the number of active modes and their positions and relative intensities. Furthermore, the model demonstrated reasonable ability to reproduce the saturation dynamics of both the laser power and the population inversion.

In addition, the model proved also to be capable of simulating the specific features of the laser frequency spectrum under the condition of homogeneously broadened gain.

Regarding the lineshape and linewidth of the individual modes, the calculation with completely inhomoge- neous gain produced a Lorentzian-like lineshape with strongly suppressed tail and a linewidth close to the spectral width of the pure cavity. The calculation with homogeneously broadened gain produced likewise a Lorentzian lineshape; however, the linewidth remained unsaturated long time after having achieved power saturation, and continued to decrease towards the calculation limit. These results strongly support the conclusion that the reason behind the very narrow linewidth reported for different kinds of lasers lies simply in the frequency competition driven by the homogeneous contribution to the overall broadening of the gain profile. And therefore, in order to allow for a direct comparison with experiment, the model has to be improved concerning first and foremost its capability to account simultaneously for both homogeneous and inhomogeneous contributions to the overall broadening of the gain profile.

\section{Acknowledgments}

This study was supported by the Atomic Energy Commission of Syria. The author would like to thank Dr. A. Mani for supporting the experimental data as well as for helpful discussions.

\section{References}

[1] A.L. Schawlow, C.H. Townes, Phys. Rev. 112, 1940 (1958).

[2] H. Haken, Laser Theory, Springer, Berlin 1984.

[3] R.D. Hempstead, M. Lax, Phys. Rev. 161, 350 (1967).

[4] H. Risken, K. Seybold, Phys. Lett. A 38, 63 (1972).

[5] H. Gerhardt, H. Welling, A. Güttner, Z. Phys. 253, 113 (1972).

[6] W.E. Lamb Jr., Phys. Rev. A 134, 1429 (1964).

[7] A.E. Siegman, Appl. Phys. Lett. 36, 412 (1980).

[8] M.O. Scully, W.E. Lamb Jr., M. Sargent III, Laser Physics, Addison-Wesley, Reading MA 1974.

[9] H.G. Charmichael, in: Lasers and Quantum Optics, Eds. L.M. Narducci, E.J. Quel, J.R. Tredicce, World Scientific, Singapore 1988, p. 52.

[10] O. Svelto, Principles of Lasers, Plenum Press, New York 1982

[11] W. Demtröder, Laser Spectroscopy, Springer, Berlin 1988.

[12] H. Statz, G. De Mars, in: Quantum Electronics, Ed. C.H. Townes, Columbia University Press, New York 1960, p. 530

[13] R. Dunsmuir, J. Electron. Control 10, 453 (1961).

[14] M. Sargent, III, M.O. Scully, in: Laser Handbook, North-Holland Publ. Co., Amsterdam 1972, p. 45

[15] R.A. Keller, E.F. Zalewski, N.C. Peterson, J. Opt. Soc. Am. 62, 319 (1972).

[16] T.W. Hänsch, A.L. Schawlow, P.E. Toschek, IEEE J. Quant. Electron. QE-8, 802 (1972).

[17] I. Burak, P.L. Houston, D.J. Sutton, J.I. Steinfeld, IEEE J. Quant. Electron. QE-7, 73 (1971). 
[18] W.W. Rigrod, J. Appl. Phys. 36, 2487 (1965).

[19] Y.J. Kaufman, U.P. Oppenheim, Appl. Opt. 13, 374 (1974).

[20] R.P. Feynman, Quantum Electrodynamics, Benjamin/ Cummings Publ. Co., California 1961.

[21] L. Mandel, Rev. Mod. Phys. 71, S274 (1999).

[22] C.K. Rhodes, A. Szöke, in: Laser Handbook, Eds. F.T. Arecchi, E.O. Schultz-DuBois, North-Holland Publ. Co, Amsterdam 1972, p. 265.
[23] J.R. Fuhr, W.L. Wiese, in: CRC Handbook of Chemistry and Physics, Ed. D.R. Lide, CRC Press, Florida 1998.

[24] W.T. Silfvast, Laser Fundamentals, Cambridge University Press, Cambridge 2004.

[25] A.E. Siegman, R. Arrathoon, Phys. Rev. Lett. 20, 901 (1968). 